Anneke C. Muller Kobold*, Ido P. Kema, Janneke Dijck-Brouwer, Karin Koerts, Michiel Weening, Twan Storteboom, Jenny E. Kootstra-Ros, Martijn van Faassen and Ineke J. Riphagen

\title{
Pepsin pretreatment corrects underestimation of 25-hydroxyvitamin D measurement by an automated immunoassay in subjects with high vitamin $\mathrm{D}$ binding protein levels
}

https://doi.org/10.1515/cclm-2021-0722

Received June 22, 2021; accepted August 17, 2021; published online September 1, 2021

Keywords: immunoassay; liquid chromatography-tandem mass spectrometry (LC-MS/MS); pepsin digestion; vitamin $\mathrm{D}$; vitamin D-binding protein (VDBP).

To the Editor,

Vitamin D is well known for its health benefits. The majority of circulating 25-hydroxyvitamin $\mathrm{D}(25(\mathrm{OH}) \mathrm{D})$ is bound with high avidity to vitamin D-binding protein (VDBP). For reliable 25(OH)D measurement, complete displacement of 25(OH)D from VDBP is essential. This is usually achieved by protein precipitation with organic solvents [1], as is often used in combination with liquid chromatography-tandem mass spectrometry (LC-MS/MS) methods. Since this pretreatment is incompatible with the use of antibodies in immunoassays, many (semi-automated) strategies have been developed to release 25(OH)D from VDBP before analysis by immunoassay [2].

Many 25(OH)D immunoassays, however, still suffer from a lack of accuracy in samples containing high levels

\footnotetext{
*Corresponding author: Anneke C. Muller Kobold, Department of Laboratory Medicine, University of Groningen, University Medical Center Groningen, Groningen, The Netherlands,

E-mail: a.c.muller@umcg.nl. https://orcid.org/0000-0003-34574179

Ido P. Kema, Janneke Dijck-Brouwer, Karin Koerts, Michiel Weening, Twan Storteboom, Jenny E. Kootstra-Ros, Martijn van Faassen and Ineke J. Riphagen, Department of Laboratory Medicine, University of Groningen, University Medical Center Groningen, Groningen, The Netherlands. https://orcid.org/0000-0002-9142-6263 (M. van Faassen)
}

of VDBP as was shown by Heijboer et al. [3]. Fujirebio developed an automated two-step non-competitive 25(OH)D immunoassay method (Lumipulse G600II) that uses a novel steroid compound to extract 25(OH)D from VDBP, which is suggested to allow for more efficient separation of 25(OH)D from VDBP [4]. This Lumipulse G immunoassay showed good correlations with Vitamin D Standardization Program (VDSP) traceable LC-MS/MS methods in healthy individuals [5-7]. However, agreement with VDSP traceable LC-MS/MS methods was moderate to poor in other populations such as pregnant women [7], and 25(OH)D concentrations were underestimated with 11$35 \%$, depending on the patients tested $[7,8]$.

We investigated whether we could correct for the underestimation of 25(OH)D in subjects with high VDBP concentrations using pepsin pretreatment in combination with the Lumipulse G 25(OH)D automated immunoassay. As the pepsin proteolytic activity can be stopped effectively by neutralizing the sample $\mathrm{pH}$, this procedure can be combined with subsequent use of antibodies in an immunoassay format.

Residual EDTA-plasma samples were collected from healthy subjects $(n=26)$, dialysis $(n=20)$, liver fibrosis $(\mathrm{n}=20)$, and ICU patients $(\mathrm{n}=20)$, and 20 and 36 weeks pregnant women $(n=37)$. All samples were anonymized before analysis. According to the Dutch Medical Research Involving Human Subjects Act no Institutional Review Board approval was required.

Concentrations of $25(\mathrm{OH}) \mathrm{D}$ were measured using an automated immunoassay (Lumipulse G600II, Fujirebio Inc., Tokyo, Japan) and a LC-MS/MS method that was calibrated using National Institute of Standards and Technology (NIST) standard reference material 2972 (as described previously [9] with intra-assay imprecision of 7.2, 5.0 and $6.3 \%$ at levels of 22, 53 and $145 \mathrm{nmol} / \mathrm{L}$, respectively, and a $\mathrm{LOQ}$ of $4 \mathrm{nmol} / \mathrm{L})$. Separation of 25(OH)D from 
VDBP and other carrier proteins was performed by pepsin digestion. In short, $20 \mu \mathrm{L}$ pepsin $0.4 \%(\mathrm{w} / \mathrm{v})$ in $1 \mathrm{M} \mathrm{HCl}$ was added to $200 \mu \mathrm{L}$ plasma and incubated for $30 \mathrm{~min}$ at room temperature. VDBP levels were measured using an enzyme linked immunosorbent assay (Immundiagnostik AG, Bensheim, Germany). Agreement between assays within patient groups was evaluated using Passing and Bablok regression analysis.

The effect of protein digestion on 25(OH)D measurement in samples with increasing VDBP levels was investigated as follows: two different samples with VDBP levels of 161 and $1482 \mathrm{mg} / \mathrm{L}$ were mixed in ratio's ranging from 1:64 to 64:1. This resulted in nine different newly formed samples with increasing VDBP levels. These nine different samples were measured using the automated immunoassay, with and without pre-treatment with pepsin, and the LC-MS/MS method. Only before immunoassay analysis, but not before LC-MS/MS analysis, $16 \mu \mathrm{L} 1 \mathrm{M} \mathrm{NaOH}$ was added to neutralize $\mathrm{pH}$. Results were corrected for dilution afterwards. Analyses were performed using Analyse-it (Excel) and GraphPad Prism (version 8.4.2). Differences in VDBP levels between groups were evaluated using Kruskal-Wallis analysis.

Assay agreement of the Lumipulse immunoassay with our LC-MS/MS method was good in most subject groups (with a slope, intercept $(\mathrm{nmol} / \mathrm{L}$ ) and correlation coefficient r of $0.87,-0.76$, and 0.98 in healthy subjects; $0.90,2.1$, and 0.99 in dialysis patients; $0.82,4.9$, and 0.98 in liver fibrosis patients; and $0.87,2.77$, and 0.87 in ICU patients, respectively), with the exception of pregnant women (Figure 1A and B). The Lumipulse assay underestimated 25(OH)D concentrations with $15 \%$ compared to the LC-MS/MS method. This effect was more pronounced (i.e., 30-35\% underestimation) in pregnant women, who had higher VDBP concentrations (i.e., median VDBP $926.3 \mathrm{mg} / \mathrm{L}$ in 36 weeks pregnant women vs. $427.5 \mathrm{mg} / \mathrm{L}$ in all subjects without pregnant women $(\mathrm{p}<0.0001)$.
The effect of pepsin digestion and subsequent pepsin neutralization on $25(\mathrm{OH}) \mathrm{D}$ measurement with the Lumipulse immunoassay compared to LC-MS/MS for different levels of VDBP is shown in Figure 2. We found an increasing discrepancy between the Lumipulse immunoassay and LC-MS/MS method with increasing levels of VDBP. Pepsin pretreatment and subsequent neutralization abolished this effect almost completely.

In this study, we demonstrated that, even at VDBP levels around $500 \mathrm{mg} / \mathrm{L}$ as seen in healthy subjects, the Lumipulse immunoassay underestimated 25(OH)D concentrations with approximately $15 \%$. This underestimation increased with increasing VDBP levels to an underestimation of $30-35 \%$ in pregnant women, who have high VDBP levels. These findings are in line with results of Cavalier et al. [7] and Giuliani et al. [8], which demonstrated that 25(OH)D concentrations were underestimated between 11 and 35\% depending on the population tested.

Moreover, we found an increasing discrepancy between the Lumipulse immunoassay and LC-MS/MS method with increasing levels of VDBP. Furthermore, we demonstrated that pepsin pretreatment and subsequent neutralization abolished this effect almost completely, indicating

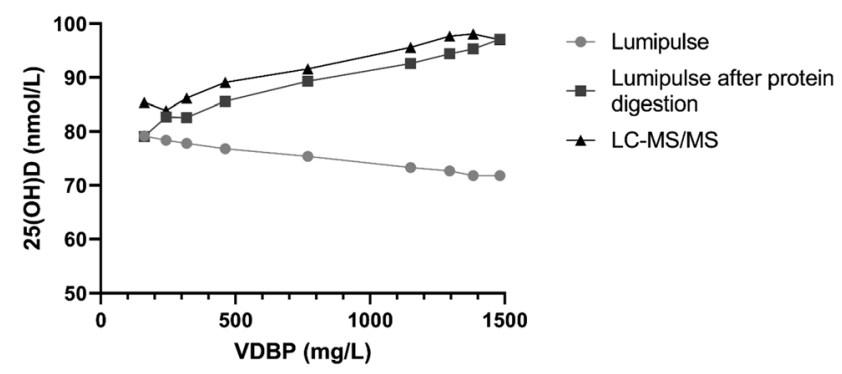

Figure 2: Effect of pepsin digestion and subsequent pepsin neutralization on $25(\mathrm{OH}) \mathrm{D}$ measurement with the Lumipulse immunoassay compared to LC-MS/MS in samples with different VDBP levels.
A

Overall w/o pregnant women

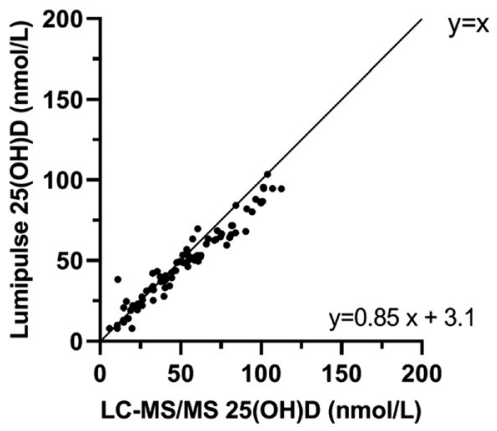

B

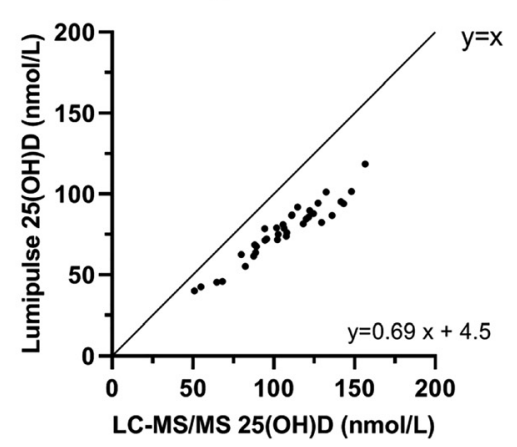

Figure 1: Method comparison of the automated Lumipulse G600II 25(OH)D immunoassay with an LC-MS/MS 25(OH)D method in all subjects except pregnant women (A) and 36 week pregnant women (B). Results of the Lumipulse G600II 25(OH)D immunoassay are obtained according to manufacturer's instructions (without pepsin pretreatment). Results of the LC-MS/MS 25(OH)D method are obtained according to the in-house Standard Operating Procedure (with pepsin pretreatment). 
that 25(OH)D was incompletely released from VDBP and other carrier proteins in subjects with high circulating VDBP concentrations before analysis with this automated immunoassay. Thus, a relative simple pretreatment of these samples using pepsin digestion and subsequent $\mathrm{pH}$ neutralization can circumvent the underestimation by 25(OH)D immunoassays.

Research funding: None declared.

Author contributions: All authors have accepted responsibility for the entire content of this manuscript and approved its submission.

Competing interests: Authors state no conflict of interest. Informed consent: Informed consent was obtained from all individuals included in this study.

Ethical approval: According to the Dutch Medical Research Involving Human Subjects Act no Institutional Review Board approval was required.

\section{References}

1. Vogeser M. Quantification of circulating 25-hydroxyvitamin D by liquid chromatography-tandem mass spectrometry. J Steroid Biochem Mol Biol 2010;121:565-73.

2. Wallace AM, Gibson S, de la Hunty A, Lamberg-Allardt C, Ashwell M. Measurement of 25 -hydroxyvitamin $D$ in the clinical laboratory: current procedures, performance characteristics and limitations. Steroids 2010;75:477-88.

3. Heijboer AC, Blankenstein MA, Kema IP, Buijs MM. Accuracy of 6 routine 25 -hydroxyvitamin $D$ assays: influence of vitamin $D$ binding protein concentration. Clin Chem 2012;58:543-8.

4. Saleh L, Mueller D, von Eckardstein A. Analytical and clinical performance of the new Fujirebio 25-OH vitamin D assay, a comparison with liquid chromatography-tandem mass spectrometry (LC-MS/MS) and three other automated assays. Clin Chem Lab Med 2016;54:617-25.

5. Wise SA, Camara JE, Sempos CT, Lukas P, Le Goff C, Peeters S, et al. Vitamin D Standardization Program (VDSP) intralaboratory study for the assessment of 25-hydroxyvitamin D assay variability and bias. J Steroid Biochem Mol Biol 2021;212:105917.

6. Cavalier E, Fraser CG, Bhattoa HP, Heijboer AC, Makris K, Ulmer CZ, et al. Analytical performance specifications for 25-hydroxyvitamin D examinations. Nutrients 2021;13:431.

7. Cavalier E, Lukas P, Bekaert AC, Peeters S, Le Goff C, Yayo E, et al. Analytical and clinical evaluation of the new Fujirebio Lumipulse ${ }^{\circledR} G$ non-competitive assay for $25(\mathrm{OH})$-vitamin $D$ and three immunoassays for $25(\mathrm{OH}) \mathrm{D}$ in healthy subjects, osteoporotic patients, third trimester pregnant women, healthy African subjects, hemodialyzed and intensive care patients. Clin Chem Lab Med 2016;54:1347-55.

8. Giuliani S, Corvetta D, Lucchiari M, Herrmann M. Evaluation of the analytical and clinical performance of the Fujirebio Lumipulse ${ }^{\circledR} \mathrm{G}$ 25-OH vitamin D assay. Ann Clin Biochem 2018;55:302-4.

9. Dirks NF, Vesper HW, van Herwaarden AE, van den Ouweland JM, Kema IP, Krabbe JG, et al. Various calibration procedures result in optimal standardization of routinely used 25(OH)D ID-LC-MS/MS methods. Clin Chim Acta 2016;462:49-54. 